\title{
Percepción de los estudiantes de las características y comportamientos de sus profesores asociados a una enseñanza clínica efectiva
}

\author{
Students' perception about the characteristics and behaviors of their teachers linked to an \\ effective clinical teaching
Percepção estudantil das características e comportamentos de professores associados à efetividade do ensino clínico

\author{
Marcela Palacios Gutiérrez', Pilar Quiroga Lagos ${ }^{2}$ \\ ${ }^{1}$ Universidad de Concepción, Oficina de Educación Médica, Facultad de Odontología, Magíster en \\ Educación Médica para las Ciencias de la Salud, Facultad de Medicina, 96384474 móvil, \\ marpalac@udec.cl \\ ${ }^{2}$ Universidad de Concepción, Departamento de Psiquiatría y Salud Mental, Departamento de Educación \\ Médica, Facultad de Medicina.
}

\begin{abstract}
RESUMEN
Para diagnosticar el estado de la enseñanza clínica en la Facultad de Odontología de la Universidad de Concepción, se evaluaron las características y comportamientos del profesor asociados a su efectividad. Esta evaluación se realizó a fines del año académico 2009 a través de cuestionarios aplicados a alumnos de cuarto y quinto año, que calificaron a sus profesores mediante un formulario de 34 ítem. Los resultados permitieron calificar la enseñanza clínica $(4,40 \mathrm{DE}=0,99)$, y describir los niveles de aprobación $(85,2 \%)$ y logro $(75,0 \%)$ para las dimensiones de dicha enseñanza definidas para este estudio. Si bien el profesor eficaz para este escenario ha sido descrito y definido en su rol, no todos los docentes llenan a cabalidad este ideal. El diagnóstico descriptivo de la enseñanza clínica, fundamentado en la evaluación de características y comportamientos del profesor, posibilita conocer el perfil del grupo evaluado, identificando sus fortalezas y debilidades.

Palabras clave: educación odontológica, enseñanza clínica, efectividad de la enseñanza, evaluación de la enseñanza, comportamientos del profesor.
\end{abstract}

\begin{abstract}
To diagnose the state of clinical teaching in the Faculty of Dentistry of the University of Concepción, teachers' characteristics and behaviors associated with its effectiveness were evaluated. This evaluation was conducted at the end of the academic year 2009, through questionnaires applied to students in fourth and fifth year, which rated their teachers on a 34-item evaluation form. The results allowed to assess the clinical teaching $(4.40 \mathrm{SD}=0.99)$, and describe the levels of approval (85.2\%) and achievement $(75.0 \%)$ for the teaching dimensions defined for this study. While an effective teacher for this scenario has been described and defined in his/her role, not all teachers fulfill this ideal. The descriptive diagnosis of clinical teaching, based on the evaluation of teacher's characteristics and behavior enables to know the profile of the group evaluated, identifying their strengths and weaknesses.

Key words: dental education, clinical teaching, teaching effectiveness, teaching assessment, teacher's behavior.

\section{RESUMO}

Para diagnosticar o estado do ensino clínico na Faculdade de Odontologia da Universidade de Concepción, avaliaram-se as características e comportamentos associados à eficácia do professor. A avaliação ocorreu no final do ano letivo de 2009, por meio da aplicação de questionários a estudantes de quarto e quinto anos. Estes classificaram as características de seus professores por meio de formulário de 34 itens. Por meio dos resultados, avaliou-se o ensino clínico $(4,40 \mathrm{DP}=0,99)$, descreveram-se os níveis de aprovação $(85,2 \%)$ e êxito $(75,0 \%)$, conforme as dimensões do ensino definidas para o estudo. Embora o professor eficaz, segundo os critérios elencados, fora descrito e definido
\end{abstract}


por suas funções, nem todos eles preencheram os requisitos para sê-lo. O diagnóstico descritivo do ensino clínico, fundamentado na avaliação das características e comportamentos docentes, permitiu conhecer o perfil do grupo avaliado, identificando seus pontos fortes e fracos.

Palavras chave: educação odontológica, ensino clínico, efetividade de ensino, avaliação do ensino, comportamentos docentes.

\section{INTRODUCCIÓN}

La enseñanza clínica en la Facultad de Odontología de la Universidad de Concepción constituye el centro de la formación de los estudiantes en el cuarto y quinto año de la carrera; en esta instancia se espera que desarrollen a cabalidad los fundamentos teóricos, habilidades y destrezas clínicas de la profesión. El modelo de enseñanza se caracteriza por la tutoría directa de un profesor para un número variable de estudiantes, dentro de un sistema disciplinar con tutores dedicados a la enseñanza en cada una de las áreas de especialidad de la odontología.

La evaluación del desempeño de estos profesores se aborda a nivel institucional dentro de un formato que no involucra los aspectos propios de la enseñanza en clínica, por lo que su valor como una instancia para diagnosticar calidad y eficacia es escaso, y tanto profesores como estudiantes no reciben los beneficios de una evaluación diseñada para este escenario.

\subsection{ENSEÑANZA EN EL AMBIENTE CLÍNICO}

La enseñanza clínica, esto es, enseñar y aprender enfocándose en, y usualmente involucrando directamente a, los pacientes y sus problemas, constituye el corazón de la educación en el área de la salud (Spencer, 2003). El ambiente de aprendizaje clínico se define como una red interactiva dentro del escenario clínico que influencia los aprendizajes de los estudiantes (Dunn \& Burnett, 1995 citado en Chan, 2003).

El aprendizaje en este ambiente tiene muchas fortalezas, a saber: se enfoca en problemas reales en el contexto del ejercicio profesional; los estudiantes son motivados por la relevancia y la participación en la resolución de los mismos; el modo de pensar, comportamiento y actitudes son modeladas por los profesores; y es el único escenario donde se puede enseñar, aprender e integrar como un todo las destrezas profesionales (Spencer, 2003).

Se pueden distinguir cuatro componentes en las experiencias clínicas de los estudiantes: las oportunidades clínicas de aprendizaje, la participación en actividades de aprendizaje específicas, la interacción con los instructores y las percepciones personales acerca de la educación clínica (Henzi et al., 2006). Martin Fugill describió tres categorías de elementos dentro de la interacción estudiante-profesor; éstas son: los comportamientos del estudiante, las características del profesor y los comportamientos de enseñanza-aprendizaje (Fugill, 2005).

Una enseñanza clínica típica involucra la supervisión del estudiante en formación por parte de un clínico con mayor experiencia, y por defecto implica una gran variedad de estilos de enseñanza (Gerzina et al., 2005). La supervisión clínica se entiende como la acción de proporcionar control, guía y retroalimentación en lo referido al desarrollo personal, profesional y educacional en el contexto de la atención de pacientes (Kilminster et al., 2007). 
En el ambiente clínico una parte importante del aprendizaje se obtiene desde el ejemplo entregado personalmente por los profesores, quienes influencian significativamente a los estudiantes; el profesor debería modelar o ejemplificar lo que éstos deben aprender. Los estudiantes aprenden por observación e imitación de los profesores a los que respetan, no sólo de lo que sus profesores les dicen, si no de lo que éstos efectivamente hacen en la práctica clínica, y de los conocimientos, destrezas y actitudes que exhiben (Harden \& Crosby, 2000; Stalmeijer et al., 2008).

Se ha sugerido que los profesores con más experiencia, que manejan las materias clínicas y pedagógicas, adquieren progresivamente en el curso de su carrera académica un conjunto tácito de conocimientos de los principios básicos de pedagogía. Peter McLeod et al. (2004) identificaron estos principios básicos, que deberían formar parte del armamento del profesor clínico; destacando las siguientes áreas: evaluación, herramientas para el aprendizaje de adultos, características del aprendizaje de adultos y currículo.

En un sentido similar, John Sweet et al. (2008) identificaron los requerimientos para una enseñanza clínica efectiva, destacando una buena práctica educacional, en la cual el profesor entiende como los estudiantes aprenden a fin de apoyar este aprendizaje; una buena logística y formación del profesor, a fin de facilitar el desarrollo educacional; y una buena organización clínica (Sweet et al., 2008).

Así, el profesor debería progresar y crecer en su formación como clínico y educador, y en este proceso incrementar su efectividad. La adaptación del modelo de 3-círculos de logros de aprendizaje de Dundee describe al profesor avanzando desde competencias técnicas a meta-competencias; el círculo interno-básico define las tareas que todo profesor clínico debe cumplir, "haciendo lo correcto"; el círculo intermedio corresponde al enfoque con el que el profesor enfrenta la enseñanza, "haciendo las cosas bien"; y el círculo exterior-avanzado se refiere al profesor como un profesional de la educación, "la persona correcta en acción" (Hesketh et al., 2001; Ramani \& Leinster, 2008).

\subsection{EVALUACIÓN DE LA EFECTIVIDAD DE LA}

ENSEÑANZA CLÍNICA Y DEL PROFESOR CLÍNICO EFECTIVO

En términos educativos una enseñanza efectiva se define en la medida que las actividades de enseñanza cumplan con el propósito, función y meta que se pretende de ellas (Jahangiri et al., 2008). El objetivo de la enseñanza clínica es proveer a los aprendices con los conocimientos, destrezas y actitudes que necesitarán en su futura vida profesional (Snell et al., 2000).

Los instrumentos que califican la efectividad de la enseñanza proporcionan tanto retroalimentación positiva como negativa a los profesores, y en condiciones ideales su finalidad es ayudar a mejorar la "habilidad para enseñar". Los resultados obtenidos en instrumentos en los cuales los estudiantes califican la efectividad de los profesores son confiables y se correlacionan con otros indicadores tales como aprendizaje del estudiante, auto-evaluación del instructor y calificación de pares (Copeland \& Hewson, 2000; Jahangiri et al., 2008).

Desde la perspectiva de los aprendices, la evaluación de la enseñanza clínica puede considerar la satisfacción con el proceso de enseñanza y sus percepciones acerca de su efectividad (Snell et al., 2000). Kristin Victoroff \& Sarah Hogan describieron las 
experiencias de aprendizaje efectivas, según la percepción de los estudiantes, como el resultado de la suma de las características y comportamientos del instructor clínico, las características del proceso de aprendizaje y del ambiente de aprendizaje (Victoroff \& Hogan, 2006).

En principio la competencia profesional y el ser un modelo a imitar, la habilidad para traspasar los conocimientos a la clínica explicando de manera sencilla los conceptos más difíciles, las habilidades comunicacionales y una actitud motivadora, la crítica constructiva y una actitud cortés, la cercanía y accesibilidad, el interés y comprensión para con los estudiantes, la puntualidad, el sentido práctico, el exhibir interés y gusto por enseñar, la consistencia e imparcialidad en el trato y al calificar, y el respeto en la relación con estudiantes y/o pacientes, han sido descritos por estudiantes de odontología como comportamientos positivos de los profesores (Fugill, 2005; Henzi et al.,2006; McGrath et al., 2005; Schönwetter et al., 2006).

Estudios realizados en educación superior que correlacionan condiciones de la enseñanza y comportamientos del profesor con indicadores como son el logro de los estudiantes, la evaluación general de la calidad de la enseñanza y la importancia otorgada por estudiantes y profesores a dichas condiciones y comportamientos, permiten reconocer el impacto que las distintas dimensiones tienen según el indicador elegido. Kenneth Feldman, correlacionó diversas dimensiones de una enseñanza efectiva con el logro de los estudiantes, determinando tanto las condiciones de enseñanza como los comportamientos del profesor que se asocian más fuertemente con el aprendizaje del estudiante. Listadas en orden jerárquico son: i) la preparación del profesor y organización del curso; ii) la claridad y comprensibilidad del profesor; iii) el impacto de la formación o logro percibido por el estudiante; iv) la estimulación por parte del profesor del interés en el curso y sus materias; v) el alentar el cuestionamiento y la discusión, y la apertura a la opinión de otros; vi) la disponibilidad y disposición para ayudar del profesor; vii) las destrezas comunicacionales y expresividad; viii) la claridad en los objetivos y requerimientos del curso; ix) el conocimiento de las materias por parte del profesor; $x$ ) la sensibilidad y preocupación con el nivel de la clase y su progreso; xi) el entusiasmo del profesor por las materias y por enseñar; xii) la justicia e imparcialidad del profesor al evaluar y la calidad de las evaluaciones; xiii) el desafío intelectual y promoción del pensamiento independiente, ya sea impulsados por el profesor o por el curso; xiv) la preocupación, respeto y amabilidad del profesor para con los estudiantes; xv) la naturaleza, calidad y frecuencia de la retroalimentación; xvi) la naturaleza y valor de las materias del curso (incluyendo su utilidad y relevancia); y xvii) la naturaleza y utilidad de materiales suplementarios y ayudas para la enseñanza (Feldman, 1989).

Si bien estos hallazgos se registran en áreas distintas a la educación odontológica, sus resultados han sido corroborados para la enseñanza clínica en odontología. Dieter Schönwetter et al. (2006) identificaron siete características principales para esta enseñanza que se correlacionan con las categorías identificadas por Feldman, a destacar, y en orden jerárquico: i) la compenetración del profesor; ii) la organización del profesor y del curso; iii) el entusiasmo del profesor; iv) los conocimientos y destrezas para enseñar; v) las características de las evaluaciones y tareas asignadas; vi) la naturaleza de la interacción grupal; y vii) la amplitud o campo de las materias abarcado por el profesor (Schönwetter et al., 2006). 
Es importante señalar que, en términos generales, existe acuerdo entre estudiantes y profesores acerca de los aspectos relevantes para una instrucción efectiva, aun cuando se registren diferencias en la importancia relativa que se otorga a éstos (Feldman 1989; Chambers et al., 2004; Gerzina et al., 2005).

Por otra parte, al comparar grupos de estudiantes con distintos niveles de formación, se encuentran diferencias en el valor otorgado por los estudiantes a las distintas dimensiones del aprendizaje clínico, y a los comportamientos y características que las componen, (Paukert \& Richards, 2000). Estos hallazgos están de acuerdo con la Teoría de Vector de Aprendizaje de Stritter que sugiere que los estudiantes progresan de la dependencia del profesor a la colaboración con éste, y de ahí a una posición de independencia para buscar sus propios aprendizajes (Stritter, 1988 citado en Paukert \& Richards, 2000); así, en cada nivel de formación el estudiante necesita de un profesor que posea determinados atributos o comportamientos.

Considerando que las condiciones de una enseñanza clínica efectiva y las características y comportamientos del profesor ideal en este escenario ya han sido descritas en la literatura, el objetivo de la investigación fue describir la enseñanza clínica en la Facultad de Odontología de la Universidad de Concepción, utilizando como fuente de información la percepción de los estudiantes acerca de las características y comportamientos de sus profesores asociados a una enseñanza efectiva.

\section{MATERIALES Y MÉTODOS}

La presente investigación se diseñó como un estudio de corte transversal para análisis descriptivo. La población del estudio como evaluador correspondió a los estudiantes cursando el 8avo y 10mo semestre en la carrera de Odontología de la Universidad de Concepción, año 2009; el universo de evaluados correspondió a los profesores de las asignaturas clínicas de operatoria, prótesis fija, prótesis removible, endodoncia, periodoncia y odontopediatría, que fueran docentes directos de los estudiantes. La muestra en ambos grupos se obtuvo por participación voluntaria, excluyendo a conveniencia los estudiantes que no cursaban la totalidad de las asignaturas clínicas del semestre o las cursaban por segunda vez.

El número mínimo de cuestionarios a aplicar se obtuvo utilizando como antecedente el valor promedio de la calificación para comportamientos de enseñanza efectiva informado por Liesel Copeland \& Mariana Hewson (4,12 DE=0,772), con seguridad de 95\% y error de muestreo no superior a 3\% de dicho valor (Copeland \& Hewson, 2000). El mismo criterio se utilizó para calcular el número mínimo de evaluadores por profesor, asegurando una participación de estudiantes de ambos niveles cuando correspondiera.

Los estudiantes participantes fueron citados a dos rondas para evaluación, una por nivel -4to y 5to año. Cada estudiante contestó tantos cuestionarios como profesores clínicos del grupo de participantes le correspondieran, con máximo posible de 7 .

\subsection{INSTRUMENTO DE EVALUACIÓN}

Para confeccionar un instrumento de evaluación ad hoc se utilizó como fundamentación el estudio de Kenneth Feldman (Feldman, 1989) que identifica y jerarquiza las 
condiciones de enseñanza y los comportamientos del profesor más fuertemente ligadas con el aprendizaje del estudiante ( medida de la efectividad del proceso de enseñanza); seleccionando aquellas dimensiones que eran aplicables a un ambiente de enseñanza clínico y que habían sido informadas como válidas en odontología (Fugill, 2005; McGrath et al., 2005; Henzi et al., 2006; Schönwetter et al.,2006,).

Se incluyeron 15 de las condiciones de enseñanza y comportamientos del profesor según Feldman, con 27 ítem para evaluación, y 7 ítem de evaluación anexos, recurrentes en los informes de enseñanza en odontología, para un total de 34 en el formato de evaluación a responder por los estudiantes. Cada ítem designado como "atributos y conductas del profesor clínico" podía ser calificado en escala de 5 puntos, siendo 5-total acuerdo, 4-acuerdo parcial, 3-ni acuerdo ni desacuerdo, 2-desacuerdo parcial, y 1-en desacuerdo. Los ítem se diseñaron para representar un comportamiento, o bien, de ser pertinente, la característica del profesor y el comportamiento exhibido correspondiente. Además se dispuso de una sección para comentarios, para la recolección de información anexa que los estudiantes consideraran importante para el aprendizaje en clínica. El formulario fue revisado para forma y comprensibilidad por profesores clínicos de odontología. La validez del constructo se determinó pos-aplicación mediante prueba de suma de rangos de Wilcoxon para el grupo de profesores con alumnos evaluadores en ambos niveles. Esta prueba corrobora validez al confirmar la hipótesis de nulidad: "no existen diferencias en las calificaciones del grupo de profesores evaluados por dos grupos de estudiantes".

La descripción de cada ítem y su agrupación en acuerdo con Feldman, si aplica, se detalla en la Figura 1. El orden del listado corresponde al que fue presentado a los estudiantes.

\begin{tabular}{|c|c|c|}
\hline Ítem & Dimensión de la enseñanza & Atributos y conductas del profesor clínico \\
\hline 1 & Organización & $\begin{array}{l}\text { Es puntual y está disponible en la clínica } \\
\text { durante las horas programadas. }\end{array}$ \\
\hline 2 & Organización & $\begin{array}{l}\text { Es organizado en sus actividades, los } \\
\text { estudiantes no tienen que esperarlo. }\end{array}$ \\
\hline 3 & $\begin{array}{c}\text { Fomento del cuestionamiento } \\
\text { y discusión y apertura a la } \\
\text { opinión de otros } \\
\end{array}$ & $\begin{array}{c}\text { Es flexible y abierto a diferentes puntos } \\
\text { de vista, ya sean de los estudiantes u otros } \\
\text { profesores. }\end{array}$ \\
\hline 4 & $\begin{array}{c}\text { Disponibilidad y disposición } \\
\text { para ayudar }\end{array}$ & Es trabajador, va más allá de sus obligaciones. \\
\hline 5 & $\begin{array}{c}\text { Fomento del cuestionamiento } \\
\text { y discusión y apertura a la } \\
\text { opinión de otros } \\
\end{array}$ & $\begin{array}{l}\text { Es capaz de criticar abiertamente y con } \\
\text { franqueza su propio desempeño. }\end{array}$ \\
\hline 6 & $\begin{array}{l}\text { Destrezas comunicacionales } \\
\text { (expresividad) }\end{array}$ & $\begin{array}{c}\text { Es expresivo, tanto en su lenguaje verbal como } \\
\text { no verbal. }\end{array}$ \\
\hline 7 & Conocimiento de la materia & $\begin{array}{l}\text { Es clínicamente competente, exhibe } \\
\text { conocimientos y destrezas clínicas acordes a su } \\
\text { área de especialidad. }\end{array}$ \\
\hline 8 & Equidad del profesor & $\begin{array}{c}\text { Es imparcial en el trato, atiende a todos los } \\
\text { estudiantes sin favoritismo. }\end{array}$ \\
\hline
\end{tabular}




\begin{tabular}{|c|c|c|}
\hline 9 & Respeto por los estudiantes & $\begin{array}{l}\text { Es respetuoso, no hace sentir a los estudiantes } \\
\text { estúpidos, intimidados o fuera de lugar. }\end{array}$ \\
\hline 10 & Equidad del instructor & $\begin{array}{l}\text { Sus calificaciones reflejan la calidad del trabajo } \\
\text { de los estudiantes. }\end{array}$ \\
\hline 11 & Profesionalismo & $\begin{array}{c}\text { Es un modelo a imitar como profesional, es } \\
\text { competente, se muestra interesado en los } \\
\text { estudiantes y pacientes. }\end{array}$ \\
\hline 12 & Sin descripción & $\begin{array}{l}\text { Es accesible y comunicativo, se muestra } \\
\text { interesado en llegar a conocer a los estudiantes. }\end{array}$ \\
\hline 13 & $\begin{array}{l}\text { Organización-Claridad de } \\
\text { objetivos y tareas del curso }\end{array}$ & $\begin{array}{l}\text { Comunica con claridad los comportamientos, } \\
\text { roles y desempeños que espera de los } \\
\text { estudiantes. }\end{array}$ \\
\hline 14 & Claridad y comprensibilidad & $\begin{array}{l}\text { Explica los conceptos y técnicas de manera } \\
\text { clara y resumida, considerando el nivel de los } \\
\text { estudiantes. }\end{array}$ \\
\hline 15 & Claridad y comprensibilidad & Responde a las preguntas detalladamente. \\
\hline 16 & $\begin{array}{l}\text { Logro percibido o impacto de } \\
\text { la enseñanza }\end{array}$ & $\begin{array}{c}\text { Enseña conceptos y procedimientos clínicos } \\
\text { actuales y pertinentes a la formación } \\
\text { profesional. }\end{array}$ \\
\hline 17 & $\begin{array}{l}\text { Estimulación del interés en el } \\
\text { curso y sus contenidos }\end{array}$ & $\begin{array}{c}\text { Logra que el aprendizaje sea interesante, } \\
\text { agradable y divertido. }\end{array}$ \\
\hline 18 & $\begin{array}{c}\text { Fomento del cuestionamiento } \\
\text { y discusión y apertura a la } \\
\text { opinión de otros }\end{array}$ & Escucha a los estudiantes. \\
\hline 19 & $\begin{array}{l}\text { Disponibilidad y disposición } \\
\text { para ayudar }\end{array}$ & $\begin{array}{c}\text { Orienta a los estudiantes en sus trabajos y } \\
\text { anticipa problemas potenciales, proporciona } \\
\text { consejos y sugerencias }\end{array}$ \\
\hline 20 & $\begin{array}{l}\text { Disponibilidad y disposición } \\
\text { para ayudar }\end{array}$ & $\begin{array}{c}\text { Disponible y receptivo, alienta a los estudiantes } \\
\text { a preguntar y buscar su ayuda. }\end{array}$ \\
\hline 21 & $\begin{array}{c}\text { Sensibilidad y preocupación } \\
\text { por el nivel de la clase y su } \\
\text { progreso } \\
\end{array}$ & $\begin{array}{l}\text { Considera el nivel de conocimientos y } \\
\text { experiencia de los estudiantes al enseñar. }\end{array}$ \\
\hline 22 & $\begin{array}{l}\text { Sensibilidad y preocupación } \\
\text { por el nivel de la clase y su } \\
\text { progreso } \\
\end{array}$ & $\begin{array}{c}\text { Muestra interés o exhibe una actitud positiva } \\
\text { acerca del trabajo y dificultades de los } \\
\text { estudiantes. }\end{array}$ \\
\hline 23 & $\begin{array}{c}\text { Sensibilidad y preocupación } \\
\text { por el nivel de la clase y su } \\
\text { progreso } \\
\end{array}$ & $\begin{array}{l}\text { Motiva a los estudiantes, da refuerzo positivo, } \\
\text { recorre la clínica para preguntar a cada uno } \\
\text { cómo va en su trabajo. }\end{array}$ \\
\hline 24 & $\begin{array}{l}\text { Entusiasmo por la materia y } \\
\text { por enseñar }\end{array}$ & $\begin{array}{l}\text { Disfruta enseñar, muestra interés en que los } \\
\text { estudiantes aprendan. }\end{array}$ \\
\hline 25 & Desafío intelectual & $\begin{array}{c}\text { Motiva a los estudiantes para que logren el } \\
\text { mejor desempeño posible. }\end{array}$ \\
\hline
\end{tabular}




\begin{tabular}{|c|c|c|}
\hline 26 & Respeto por los estudiantes & $\begin{array}{l}\text { Cuestiona el juicio y/o las capacidades de los } \\
\text { estudiantes en frente del paciente. }\end{array}$ \\
\hline 27 & $\begin{array}{l}\text { Retroalimentación a los } \\
\text { estudiantes }\end{array}$ & $\begin{array}{c}\text { Analiza el trabajo de los estudiantes y les dice } \\
\text { tanto lo que están haciendo correctamente y lo } \\
\text { que están haciendo incorrectamente. }\end{array}$ \\
\hline 28 & $\begin{array}{l}\text { Retroalimentación a los } \\
\text { estudiantes }\end{array}$ & $\begin{array}{l}\text { Proporciona retroalimentación frecuente y } \\
\text { constructiva, indica "como mejora" en una } \\
\text { manera positiva. }\end{array}$ \\
\hline 29 & $\begin{array}{l}\text { Retroalimentación a los } \\
\text { estudiantes }\end{array}$ & $\begin{array}{c}\text { Explica a los estudiantes los motivos por } \\
\text { los cuales sus trabajos/desempeños no son } \\
\text { aceptables. }\end{array}$ \\
\hline 30 & $\begin{array}{c}\text { Importancia de la } \\
\text { demostración, preparado para } \\
\text { enseñar }\end{array}$ & $\begin{array}{c}\text { Demuestra o ejecuta procedimientos en los } \\
\text { pacientes de los estudiantes como un modo de } \\
\text { enseñar. }\end{array}$ \\
\hline 31 & Profesionalismo & Toma en serio su trabajo como profesor. \\
\hline 32 & Autonomía del estudiante & $\begin{array}{l}\text { Permite a los estudiantes actuar tan } \\
\text { independientemente como sea posible, } \\
\text { cuidando la seguridad de pacientes y } \\
\text { estudiantes. }\end{array}$ \\
\hline 33 & $\begin{array}{l}\text { Respeto por la relación } \\
\text { estudiante/paciente }\end{array}$ & $\begin{array}{l}\text { Elogia el trabajo de los estudiantes, cuando } \\
\text { es merecido, frente a los pacientes, otros } \\
\text { profesores y/o pares. }\end{array}$ \\
\hline 34 & Sin descripción & $\begin{array}{l}\text { Crea un ambiente de aprendizaje positivo, libre } \\
\text { de tensión, siendo relajado, tolerante, calmado } \\
\text { y paciente. }\end{array}$ \\
\hline
\end{tabular}

Figura 1. Ítem atributos y conductas del profesor clínico

\subsection{ANÁLISIS DE DATOS}

Los datos obtenidos de los cuestionarios fueron traspasados para su análisis a planilla de Microsoft Excel (Microsoft Office 2007); utilizando las herramientas provistas por Microsoft Excel y por el sistema para análisis de datos IBM SPSS Statistics 19. Se calculó calificación promedio y DE para cada dimensión de la enseñanza. Para efectos de describir el nivel de aprobación (NA) y nivel de logro (NL) se establecieron los siguientes criterios de evaluación:

- Nivel de Aprobación. Representa el porcentaje de alumnos que califican las características y comportamientos de sus profesores en términos aprobatorios. Para el cálculo de este parámetro se determinó un nivel de exigencia de $61 \%$. Toda vez que los "atributos y conductas del profesor clínico" fueron calificados al menos con puntaje 4 (acuerdo parcial) se consideró como aprobado.

- Nivel de Logro. Representa el porcentaje ponderado de las calificaciones obtenidas. Para cada nivel de puntaje se asignó un valor de 2. Para el cálculo de este parámetro las calificaciones 1, 2 y 3 aportan (0) punto al estar bajo el nivel de aprobación y no 
contribuyen al logro; la calificación 4 aporta (1) punto como medianamente logrado y la calificación 5 (2) para totalmente logrado.

Este enfoque define un límite inferior o mínimo deseable representado por los valores de nivel de aprobación, y una medida que describe que tan cerca o lejos se está del profesor ideal que se obtiene desde el nivel de logro. La correlación existente entre la calificación tradicional: promedio y DE, y los indicadores utilizados en este estudio de NA y NL fue determinada mediante prueba $r$-Pearson.

- Análisis de Comentarios. Se consideraron comentarios válidos aquéllos que no fueran halagos o críticas al profesor y que no reiteraran conceptos incluidos en el cuestionario.

\section{RESULTADOS}

Del total de encuestas recolectadas se descartaron 7 por errores de forma, ítem no calificados y/o falta de identificación del evaluador (género o curso) o del evaluado (nombre para asignar código numérico), para un total de 218 unidades útiles para análisis. La distribución de profesores y estudiantes participantes se muestra en las Figuras 2, 3 y 4.

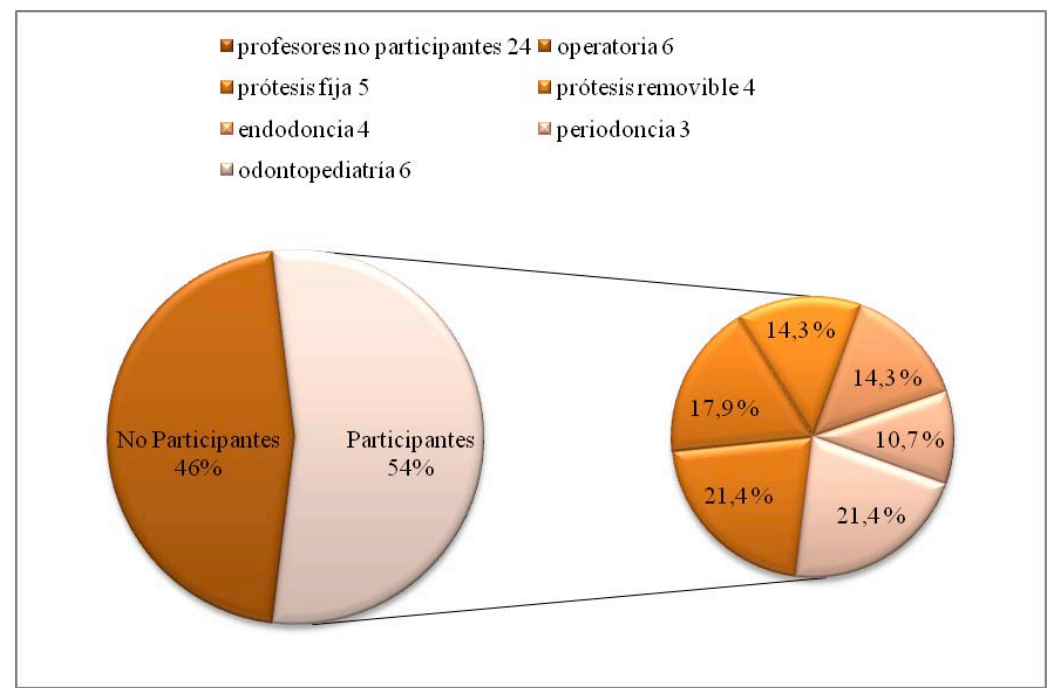

Figura 2. Participación de los profesores por disciplina clínica 


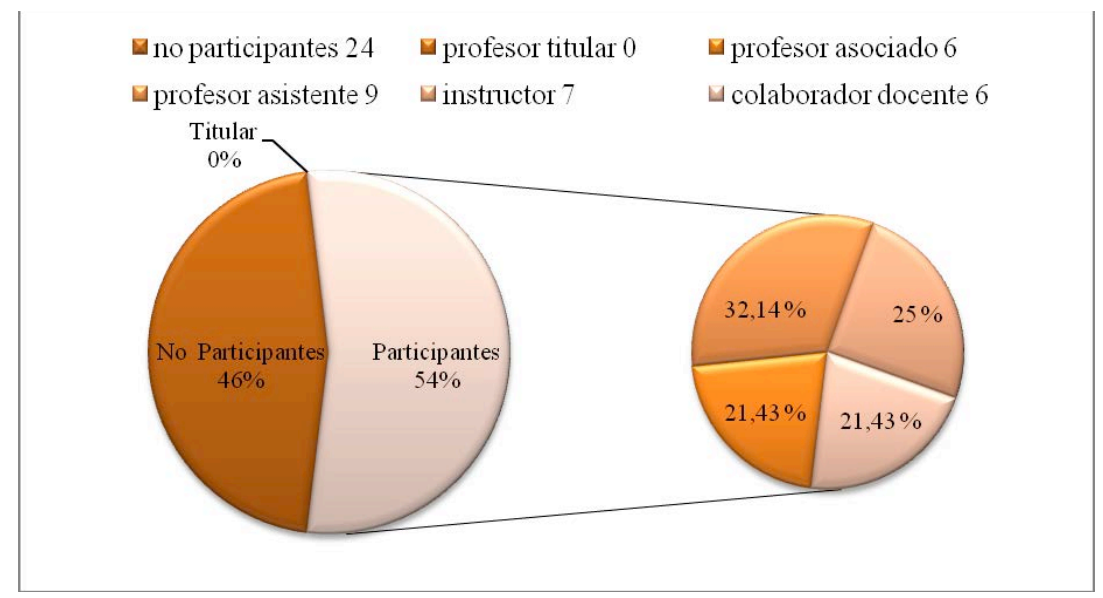

Figura 3. Participación de los profesores por jerarquía académica

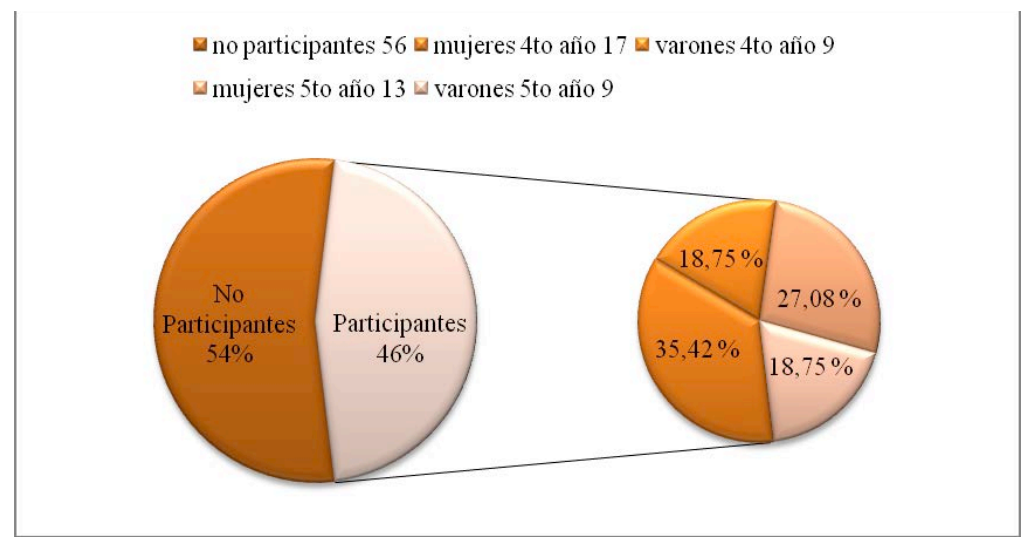

Figura 4. Participación de los estudiantes por género y nivel

Las calificaciones promedio y desviación estándar para las dimensiones de la enseñanza se presentan en la Tabla 1. Siete dimensiones se encuentran bajo la calificación global $(4,40)$ y está destacadas $(*)$. Sólo la dimensión representada por el anexo respeto por la relación estudiante-paciente califica bajo 4. Las dimensiones de la enseñanza consideradas en el estudio de Keneth Feldman están listadas en acuerdo a la correlación con logro del estudiante. 


\begin{tabular}{|c|c|c|}
\hline Dimensión de la enseñanza & $\begin{array}{c}\text { Calificación } \\
\text { promedio }\end{array}$ & DE \\
\hline Organización & $4,19^{*}$ & 1,14 \\
\hline Claridad y comprensibilidad & 4,50 & 0,90 \\
\hline Logro percibido o impacto de la enseñanza & 4,40 & 0,97 \\
\hline Estimulación del interés en el curso y sus materias & $4,30^{*}$ & 1,01 \\
\hline Fomento del cuestionamiento y discusión y apertura a la opinión & 4,40 & 0,96 \\
\hline Disponibilidad y disposición para ayudar & & \\
\hline Destrezas comunicacionales (expresividad) & $4,28^{*}$ & 1,10 \\
\hline Claridad de objetivos y tareas del curso & 4,57 & 0,80 \\
\hline Conocimiento de la materia & 4,44 & 0,89 \\
\hline Sensibilidad y preocupación por el nivel de la clase y su progreso & 4,54 & 0,94 \\
\hline Entusiasmo por la materia y por enseñar & 4,42 & 0,95 \\
\hline Equidad del instructor & 4,44 & 0,93 \\
\hline Desafío intelectual & $4,36 *$ & 1,03 \\
\hline Respeto por los estudiantes & 4,53 & 0,97 \\
\hline Retroalimentación a los estudiantes & 4,43 & 0,98 \\
\hline Anexo profesionalismo & 4,55 & 0,91 \\
\hline Anexo importancia de la demostración, preparado para enseñar & $4,16^{*}$ & 1,16 \\
\hline Anexo autonomía del estudiante & 4,81 & 0,52 \\
\hline Anexo respeto por la relación estudiante-paciente & $3,90^{*}$ & 1,11 \\
\hline Anexo ítem 12: es accesible y comunicativo & $4,30^{*}$ & 1,05 \\
\hline Calificación global $34:$ crea un ambiente de aprendizaje positivo & 4,72 & 0,74 \\
\hline $\mathbf{4 , 4 0}$ & $\mathbf{0 , 9 9}$ \\
\hline
\end{tabular}

Tabla 1. Calificación dimensiones de la enseñanza: promedio y desviación estándar

El nivel de aprobación (NA) y el nivel de logro (NL) para cada dimensión se describen en la Tabla 2. Las dimensiones que califican bajo el valor global (NA 85,2\% y NL $75,0 \%$ ) están destacadas (*). Las mayores discrepancias entre NA y NL se registran en aquellas dimensiones que registran valores más altos.

\begin{tabular}{|c|c|c|}
\hline Dimensión de la enseñanza & \% NA & \% NL \\
\hline Organización & $82,0^{*}$ & $68,1^{*}$ \\
\hline Claridad y comprensibilidad & 87,6 & 78,4 \\
\hline Logro percibido o impacto de la enseñanza & 86,2 & 75,0 \\
\hline $\begin{array}{c}\text { Estimulación del interés en el curso y sus materias } \\
\text { Fomento del cuestionamiento y discusión y apertura a la } \\
\text { opinión de otros }\end{array}$ & $81,2^{*}$ & $70,0^{*}$ \\
\hline
\end{tabular}




\begin{tabular}{|c|c|c|}
\hline Disponibilidad y disposición para ayudar & $80,9^{*}$ & $70,5^{*}$ \\
\hline Destrezas comunicacionales (expresividad) & 90,8 & 81,0 \\
\hline Claridad de objetivos y tareas del curso & 86,7 & 75,2 \\
\hline Conocimiento de la materia & 87,6 & 81,4 \\
\hline Sensibilidad y preocupación por el nivel de la clase y su \\
progreso & 87,2 & 75,2 \\
\hline Entusiasmo por la materia y por enseñar & 85,3 & 75,9 \\
\hline Equidad del instructor & 85,6 & 77,1 \\
\hline Desafío intelectual & $83,0^{*}$ & $72,2^{*}$ \\
\hline Respeto por los estudiantes & 88,8 & 81,5 \\
\hline Retroalimentación a los estudiantes & 86,9 & $74,5^{*}$ \\
\hline Anexo profesionalismo & 88,8 & 81,5 \\
\hline Anexo importancia de la demostración, preparado para & $79,8^{*}$ & $66,5^{*}$ \\
\hline enseñar & & \\
\hline Anexo autonomía del estudiante & 96,8 & 91,3 \\
\hline Anexo respeto por la relación estudiante-paciente & $67,4^{*}$ & $52,5^{*}$ \\
\hline Anexo 12: es accesible y comunicativo & $81,7^{*}$ & $70,6^{*}$ \\
\hline Anexo 34: crea un ambiente de aprendizaje positivo & 95,4 & 88,5 \\
\hline Calificación global & $\mathbf{8 5 , 2}$ & $\mathbf{7 5 , 0}$ \\
\hline
\end{tabular}

Tabla 2. Nivel de aprobación (NA) y nivel de logro (NL) para cada dimensión de la enseñanza

La existencia de una relación lineal directa y estadísticamente significativa entre los valores registrados para el delta NA-NL y el valor de DE, fue determinada por correlación r-Pearson $=0,638(\mathrm{p}<0,002$ bilateral, $\alpha=0,01)$.

Los valores de la prueba de suma de rangos de Wilcoxon para calificaciones promedio $(p=0,081, \alpha=0,05)$, nivel de aprobación $(p=0,218, \alpha=0,05)$, y nivel de logro $(p=0,433$, $\alpha=0,05)$, determinados para el grupo de profesores con evaluadores en ambos niveles, corroboraron la validez del constructo.

Los comentarios de los estudiantes no aportaron información anexa relevante que describiera otras características o comportamientos de enseñanza efectiva no consideradas en el cuestionario.

\section{DISCUSIÓN}

La evaluación de una "enseñanza clínica efectiva" es una tarea compleja y a fin de limitar el área a evaluar se debe considerar un marco teórico y conceptual que la guíe. Esta investigación se enfocó exclusivamente en las características y comportamientos del profesor clínico que se asocian a una enseñanza clínica efectiva. La relevancia de las dimensiones evaluadas se fundamentó en la asociación con logro de los estudiantes (Feldman, 1989; Schönwetter et al., 2006). 
Al ordenar las dimensiones de la enseñanza, considerando como indicador sus calificaciones para Nivel de Logro (NL) - el parámetro que mejor define la calidad de las características y comportamientos del profesor- se obtiene una jerarquización que difiere del ordenamiento original según Feldman (valores entre paréntesis). La jerarquía de logro es la siguiente:

\begin{tabular}{|c|l|}
\hline $1^{\circ}$ & Respeto por los estudiantes $\left(14^{\circ}\right)$ \\
\hline $2^{\circ}$ & Conocimiento de la materia $\left(9^{\circ}\right)$ \\
\hline $3^{\circ}$ & Destrezas comunicacionales (expresividad) $\left(7^{\circ}\right)$ \\
\hline $4^{\circ}$ & Claridad y comprensibilidad $\left(2^{\circ}\right)$ \\
\hline $5^{\circ}$ & Equidad del instructor $\left(12^{\circ}\right)$ \\
\hline $6^{\circ}$ & Entusiasmo por la materia y por enseñar $\left(11^{\circ}\right)$ \\
\hline $7^{\circ}$ & Claridad de objetivos y tareas del curso $\left(8^{\circ}\right)$ \\
\hline $7^{\circ} e$ & Sensibilidad y preocupación por el nivel de la clase y su progreso $\left(10^{\circ}\right)$ \\
\hline $9^{\circ}$ & Logro percibido o impacto de la enseñanza $\left(3^{\circ}\right)$ \\
\hline $10^{\circ}$ & Retroalimentación a los estudiantes $\left(15^{\circ}\right)$ \\
\hline $11^{\circ}$ & Fomento del cuestionamiento y discusión y apertura a la opinión de otros $\left(5^{\circ}\right)$ \\
\hline $12^{\circ}$ & Desafío intelectual $\left(13^{\circ}\right)$ \\
\hline $13^{\circ}$ & Disponibilidad y disposición para ayudar $\left(6^{\circ}\right)$ \\
\hline $14^{\circ}$ & Estimulación del interés en el curso y sus materias $\left(4^{\circ}\right)$ \\
\hline $15^{\circ}$ & Organización $\left(1^{\circ}\right)$ \\
\hline
\end{tabular}

\section{e: valor de calificación NL empatado}

Esta nueva jerarquía describe la enseñanza clínica a partir de la calificación que hacen los estudiantes de las dimensiones que la constituyen y permite reconocer fortalezas y debilidades en el escenario evaluado. Nueve dimensiones se agrupan entre los valores NL 71-79\%. Las tres primeras jerarquías califican sobre NL 80\%; de ellas, el "respeto por los estudiantes" y las "destrezas comunicacionales" se han identificado como dimensiones relevantes para la enseñanza clínica odontológica (Chambers et al., 2004; Schönwetter et al., 2006)

Respecto del "conocimiento del profesor", su calificación sobre el valor global debe considerarse dentro del contexto de las calificaciones de las otras dimensiones evaluadas, ya que se trata de una de las áreas de la enseñanza clínica que presenta mayor variabilidad en relación a la importancia asignada por estudiantes y profesores (Chambers et al., 2004; Schönwetter et al., 2006).

Una debilidad es descrita por la calificación para las dimensiones de "disponibilidad y disposición para ayudar" (NL 70,5\%), y particularmente de “organización” (NL 68,1\%), que califican al final de la jerarquía, y han sido informadas en la literatura odontológica como influyentes para una enseñanza eficaz en el escenario clínico (Henzi et al., 2005; Victoroff \& Hogan, 2006). 
Otra debilidad la describe la calificación del anexo "importancia de la demostración, preparado para enseñar" (NL 66,5\%). Esta calificación es particularmente válida si se considera que los estudiantes de odontología deben ejecutar procedimientos y adquirir un conjunto de destrezas clínicas, y la demostración de estos procedimientos por parte del profesor ayuda a preparar a los estudiantes para la práctica clínica independiente (Gerzina et al., 2005; Fugill, 2005).

\section{CONCLUSIONES}

La experiencia de aprendizaje en clínica es para los estudiantes la oportunidad para "ser dentista" en un ambiente protegido por los profesores. En esta circunstancia los docentes deben ser conscientes de su real influencia en el aprendizaje de los alumnos, y particularmente del hecho que sus características y comportamientos constituyen herramientas de enseñanza.

Si bien el profesor eficaz para este escenario ha sido descrito y definido en su rol, no se puede esperar que todos y cada uno de nuestros docentes llenen a cabalidad este ideal. La intención primaria del estudio fue realizar un diagnóstico descriptivo de la efectividad de la enseñanza clínica fundamentado en la evaluación de características y comportamientos del profesor; el resultado fue reconocer el perfil docente con las fortalezas y debilidades que influyen en dicha efectividad.

Una limitación cierta de esta investigación es que la calificación global de la enseñanza clínica describe con seguridad al grupo de profesores evaluados, pero la extrapolación al universo de profesores clínicos debe ser considerada de manera cuidadosa, ya que la motivación de los docentes para participar del estudio no fue explorada.

Ya que no todos los profesores fueron evaluados, este perfil constituye sólo una aproximación a la realidad; y la calificación global, y para las diferentes dimensiones de la enseñanza, no puede compararse con estudios realizados por otros investigadores. Sin embargo, la calificación con los conceptos edumétricos de Nivel de Aprobación y Nivel de Logro resultó de utilidad en términos descriptivos para el grupo evaluado, y en términos comparativos para otras investigaciones que describen las áreas que mejoran o deterioran la enseñanza clínica odontológica, de esta manera se evidenciaron las fortalezas y debilidades en el escenario evaluado.

Las fortalezas fueron detectadas en las áreas de "respeto por los estudiantes" y "destrezas comunicacionales"; las debilidades estuvieron en las dimensiones de "organización" y "disponibilidad y disposición para ayudar". El "conocimiento del profesor", bien evaluado en este estudio, es una dimensión reconocida como importante pero siempre en conflicto en cuanto a su peso relativo dentro del rol del profesor; nuestra impresión es que sólo adquiere valor en la medida que forme parte de un conjunto de características y comportamientos positivos.

\section{REFERENCIAS BIBLIOGRÁFICAS}

Chambers, D., Geissberger, M. \& Leknius, C. (2004). Association amongst factors thought to be important by instructors in dental education and perceived effectiveness of these instructors by students. Eur J Dent Educ, n. 8, 147-151. 
Chan, D. (2003). Validation of the clinical learning environment inventory. Western Journal of Nursing Research, n. 5, 519-532.

Copeland, H. \& Hewson, M. (2000). Developing and testing an instrument to measure the effectiveness of clinical teaching in an academic medical center. Acad Med, n. 75, 161-166.

Feldman, K. (1989). The association between student ratings of specific instructional dimensions and student achievement: refining and extending the synthesis of data from multisection validity studies. Research in Higher Education, n. 30, 583-645.

Fugill, M. (2005). Teaching and learning in dental student clinical practice. Eur J Dent Educ, n. 9, 131-136.

Gerzina, T., Mclean, T. \& Fairley, J. (2005). Dental clinical teaching: perceptions of students and teachers. J Dent Educ, n. 69, 1377-1384.

Harden, R. \& Crosby, J. (2000). AMME guide $\mathrm{n}^{\circ} 20$ : the good teacher is more than a lecturer-the twelve roles of the teacher. Med Teacher, n. 22, 334-347.

Henzi, D., Davis, E., Jasinevicius, R., Hendricson, W., Cintron, L. \& Isaacs, M. (2005). Appraisal of the dental school learning environment: the students' view. J Dent Educ, n. 69, 1137-1147.

Henzi, D., Davis, E., Jasinevicius, R. \& Hendricson, W. (2006). North American dental students' perspectives about their clinical education. $J$ Dent Educ, n. 70, 361-377.

Hesketh, E., Bagnall, G., Buckley, E., Friedman, M., Goodall, E., Harden, R. et al. (2001). A framework for developing excellence as a clinical educator. Med Educ, n. 35, 555-564.

Jahangiri, L., Mucciolo, T., Choi, M. \& Spielman, A. (2008). Assessment of teaching effectiveness in U.S. dental schools and the value of triangulation. J Dent Educ, n. 72, 707-718.

Kilminster, S., Cottrell, D., Grant, J. \& Jolly, B. (2007). AMME guide n`27: effective educational and clinical supervision. Med Teacher, n. 29, 2-19.

Mcgrath, C., Wai Kit Yeung, R., Comfort, M. \& Mcmillan A. (2005). Development and evaluation of a questionnaire to evaluate clinical dental teachers (ECDT). Br Dent J, n. 198, 45-48.

Mcleod, P., Meagher, T., Steinert, Y., Schuwirth, L. \& Mcleod, A. (2004). Clinical teachers' tacit knowledge of basic pedagogic principles. Med Teacher, n. 26, 23-27.

Paukert, J. \& Richards, B. (2000). How medical students and residents describe the roles and characteristics of their influential clinical teachers. Acad Med, n. 75, 843-845.

Ramani, S. \& Leinster, S. (2008). AMME guide $\mathrm{n}^{\circ}$ 34: teaching in the clinical environment. Med Teacher, n. 30, 347-364.

Schönwetter, D., Lavigne, S., Mazurat, R. \& Nazarko, O. (2006). Students' perceptions of effective classroom and clinical teaching in dental and dental hygiene education. $J$ Dent Educ, n. 70, 624-635.

Snell, L., Tallett, S., Haist, S., Hays, R., Norcini, J. \& Prince, K. (2000). A review of the evaluation of clinical teaching: new perspectives and challenges. Med Educ, n. 34, 862-870.

Spencer, J. (2003). ABC of learning and teaching in medicine: learning and teaching in the clinical environment. $B M J$, n. 326, 591-594.

Stalmeijer, R., Dolmans D., Wolfhagen I., Muijtjens, A. \& Scherpbier, A. (2008). The development of an instrument for evaluating clinical teachers: involving stakeholders to determine validity. Med Teacher, n. 30, 272-277.

Sweet, J., Wilson, J. \& Pugsley, L. (2008). Chairside teaching and dental perceptions of dental teachers in the UK. Br Dent J, n. 205, 565-569.

Victoroff, K. \& Hogan, S. (2006). Students' perceptions of effective learning experiences in dental school: a qualitative study using a critical incident technique. J Dent Educ, n. 70, 124-132. 
